\title{
Growth and characterization of CdSe nanoneedles and other one-dimensional CdSe nanostructures
}

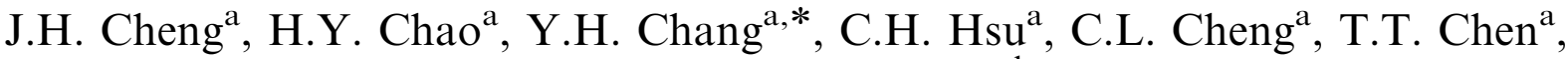 \\ Y.F. Chen ${ }^{\mathrm{a}}$, M.W. Chu ${ }^{\mathrm{b}}$ \\ ${ }^{a}$ Department of Physics, National Taiwan University, Taipei 106, Taiwan \\ ${ }^{\mathrm{b}}$ Center for Condensed Matter Sciences, National Taiwan University, Taipei 106, Taiwan
}

Available online 21 September 2007

\begin{abstract}
CdSe nanoneedles and other one-dimensional CdSe nanostructures were grown successfully by using metal organic chemical vapor deposition. These one-dimensional CdSe nanostructures could be found on the top surface as well as on the side surface of the GaAs substrate. The nanoneedles that were found to grow from the side surface could have length over $100 \mu \mathrm{m}$. Energy dispersive spectroscopy reveals that the tops of the nanoneedles grown on the side surface contain only $\mathrm{Cd}$ and $\mathrm{Se}$, no trace of the catalyst such as gold is found. From photoluminescence measurement it was found that the nanostructures grown on the top surface could have either wurtzite or zincblende crystal structures, and there are more nanostructures with wurtzite structure than that with zinc-blende structure. This conclusion was also confirmed by the X-ray diffraction.
\end{abstract}

(C) 2007 Elsevier B.V. All rights reserved.

PACS: 73.61.Ga; 73.63.Nm; 78.55.Et; 78.67.-n; 78.67.Lt

Keywords: CdSe; Nanoneedle; One-dimensional nanostructures

One-dimensional (1-D) nanostructures, such as nanowires, nanotubes, and nanobelts, have attracted plenty of interest because of their potential applications in electronics and optoelectronics [1-5]. CdSe is an important II-VI material and because of its good optical properties, the growth of 1-D CdSe nanostructures has received much attention recently. Many kinds of CdSe nanostructures, including nanobelt, nanosaw, nanopyramid, etc., have been successfully grown by using growth methods such as synthesis in a furnace, metal organic chemical vapor deposition (MOCVD), and electrodeposition. Good crystalline quality was reported for 1-D CdSe nanostructures grown with furnace synthesis and MOCVD methods by vapor-liquid-solid (VLS) mechanism [6,7], e.g., Shan et al. reported that the highly oriented nanoneedles with zincblende structure could be successfully grown at $430^{\circ} \mathrm{C}$ with an MOCVD system [8]. In this paper, we report our study

\footnotetext{
*Corresponding author. Tel.: + 886233665126 ; fax: + 886233665126 .

E-mail address: yhchang@phys.ntu.edu.tw (Y.H. Chang).
}

and characterization of the CdSe nanostructures grown by MOCVD. We found that the nanostructures grown on the top surface of the GaAs substrate at temperature $630^{\circ} \mathrm{C}$ have the mixture of wurtzite and zinc-blende structures. In addition to the nanostructures that was grown at the top surface, it was found that there were also nanoneedles grown at the side surface of the substrate. These nanoneedles were found to have length as long as $100 \mu \mathrm{m}$, and are of wurtzite structure.

$\mathrm{CdSe}$ nanostructures were grown on the $\mathrm{GaAs}\left(\begin{array}{lll}1 & 0 & 0\end{array}\right)$ substrate in MOCVD system at $630^{\circ} \mathrm{C}, 1 \mathrm{~atm}$. The precursors used were dimethylcadmium (DMCd) and dimethylselenide (DMSe). The flow rates of DMCd and DMSe were set at 25.3 and $49.6 \mu \mathrm{mole} / \mathrm{min}$ (1:2), respectively. Before putting the GaAs substrate into the quartz reactor of MOCVD system, it was coated with 1.5 to $4.5 \mathrm{~nm}$-thick sputter-coated $\mathrm{Au}$ in a sputtering system. After loading the substrate, the temperature was raised to $630^{\circ} \mathrm{C}$ and was kept at $630^{\circ} \mathrm{C}$ for 5 min to melt $\mathrm{Au}$ thin films to form clusters. The diameter of these clusters are 
about $10-20 \mathrm{~nm}$ for $1.5 \mathrm{~nm}$ Au film, $20-40 \mathrm{~nm}$ for $3 \mathrm{~nm} \mathrm{Au}$ film, and $30-50 \mathrm{~nm}$ for $4.5 \mathrm{~nm} \mathrm{Au}$ film. DMCd and DMSe shutters were then opened for $500 \mathrm{~s}$ to grow 1-D CdSe nanostructures. Scanning electron microscope (SEM) was used to observe the morphologies of the sample. Energy dispersive spectrometer (EDS) and X-ray diffraction (XRD) were studied for compositional analyses. Conventional photoluminescence (PL) and micro-PL spectra were measured by using $\mathrm{He}-\mathrm{Cd}$ laser as the excitation source to study the optical responses of these 1-D CdSe nanostructures.

The SEM images of the nanostructures, grown by using MOCVD on the GaAs substrate coated with different thickness of gold films, are shown in Fig. 1. In Fig. 1(a), the thickness of the gold film is $1.5 \mathrm{~nm}$; in Fig. 1(b), the film thickness is $3 \mathrm{~nm}$; and in Fig. 1(c), the film thickness is $4.5 \mathrm{~nm}$. In addition to the common features in the figures,
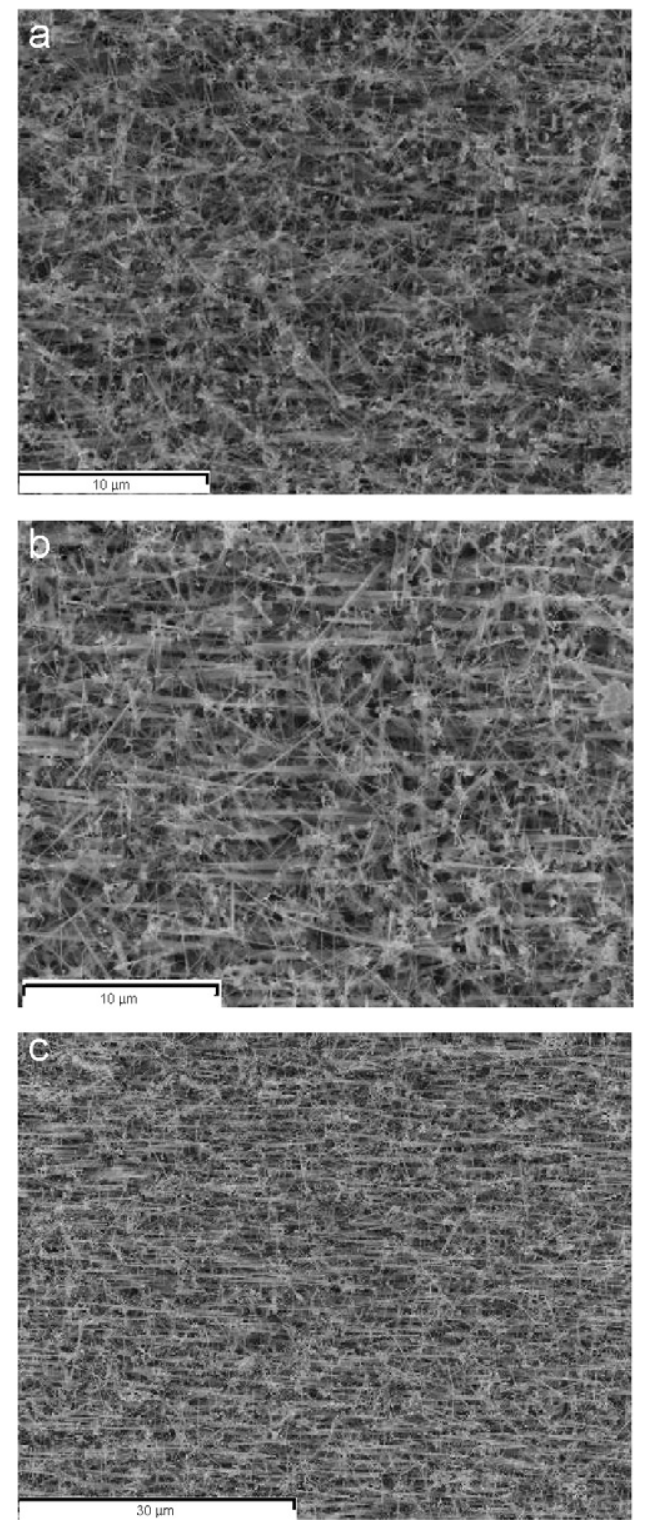

Fig. 1. SEM image of various 1-D CdSe nanostructures grown on the GaAs(1 00 ) substrate with Au film: (a) $1.5 \mathrm{~nm}$, (b) $3 \mathrm{~nm}$, (c) $4.5 \mathrm{~nm}$. i.e. there are many different kinds of nanostructures such as nanowire, nanorod, nanobelt and nanocrystal, it could be seen that as the gold film thickness gets thicker, the alignment of the nanorod gets better. This observation could be useful for growing directional CdSe nanorods which could be used for emitting polarized light. X-ray scattering was used to study the crystal structure of the CdSe nanostructures and the result of the $\theta-2 \theta$ scan is shown in Fig. 2(a). In this spectrum, diffraction peaks corresponding to the $\mathrm{CdSe}(004)$ zinc-blende structure and the $\mathrm{CdSe}(203)$ wurtzite structure could both be found. Judging from the diffraction intensity, it is found that there are more nanostructures with wurtzite structure than that with zinc-blende structure. PL experiment was employed to study the optical response of the nanostructures, and the spectrum taken at $T=15 \mathrm{~K}$ is shown in Fig. 2(b). The spectrum is asymmetrical and could be decomposed into two Gaussian curves with the peak energies corresponding to 1.78 and $1.83 \mathrm{eV}$, respectively. It is known that at low temperature, the bandgaps of CdSe with zinc-blende and wurtzite structures are 1.76 and $1.84 \mathrm{eV}$, respectively [9-12]. The two peaks in our spectrum could thus be identified as PL emission from CdSe with zinc-blende and wurtzite structures, respectively. As the relative intensity of the emission from wurtzite structure is larger than that of the emission from zinc-blende structure, it implies that there are more 1-D CdSe with wurtzite structure than 1-D CdSe with zinc-blende structure on the sample.

In addition to the nanostructures grown on the top surface of the substrate, well-aligned, dense, straight, and long CdSe nanoneedles are found to grow on the side surface of the substrate. The SEM image of these nanoneedles is shown in Fig. 3(a). It could be seen in the figure that all the nanoneedles are tens of $\mu \mathrm{m}$ long, and some of them are even over $100 \mu \mathrm{m}$. The bottoms of these nanoneedles are along the edge of the sample, and their diameters are about $5 \mu \mathrm{m}$. The growth direction of the nanoneedles is perpendicular to the direction of $\mathrm{H}_{2}$ flow and parallel to the substrate. We see that the nanoneedles stretch from the bottoms and their diameters get smaller and smaller as they get longer and longer. Micro-PL measurement was employed to investigate the properties of these nanoneedles. In doing the experiment, the laser was focused to $1 \mu \mathrm{m}$ at one of these nanoneedles. The PL spectrum taken at room temperature is shown in Fig. 3(b). The peak energy of the spectrum is at $1.74 \mathrm{eV}$ and corresponds to the bandgap energy of CdSe with wurtzite structure at room temperature [9]. The peak could be fitted with one Gaussian curve, indicating that the $\mathrm{CdSe}$ nanoneedles grown on the side surface are wurtzite structures.

It is generally accepted that in growing nanostructures, a thin Au film is needed, as the reaction needs Au, to serve as the catalyst. EDS was used to analyze the caps of the nanostructures that we grew. It was found that the caps of the nanostructures grown on the top surface contain $\mathrm{Au}$ and $\mathrm{Cd}$ when $\mathrm{Au}$ served as catalyst. However, no Au trace 
a

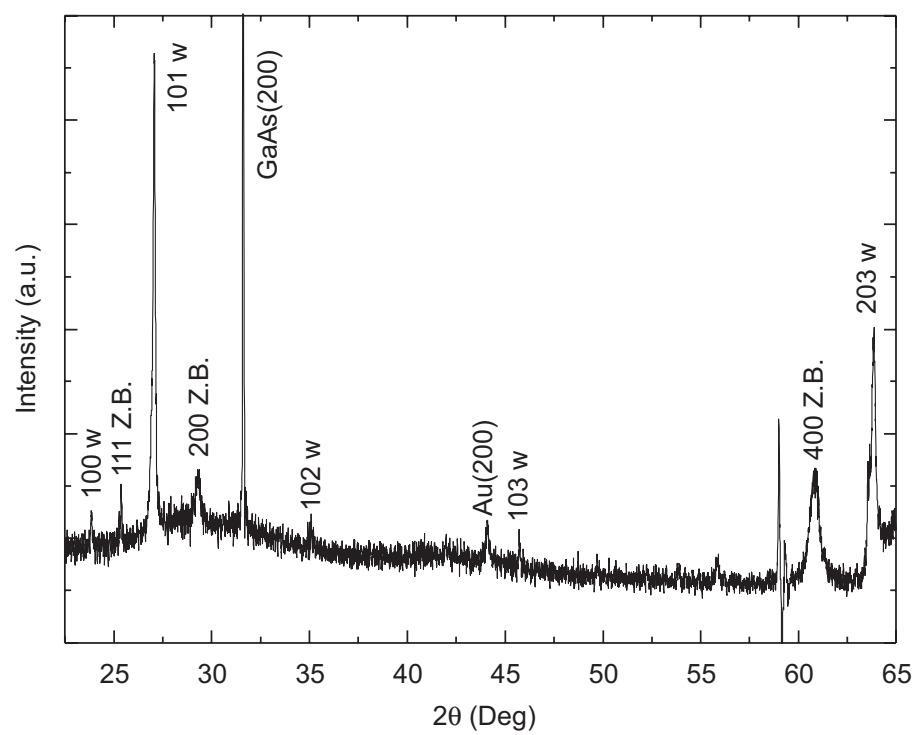

b

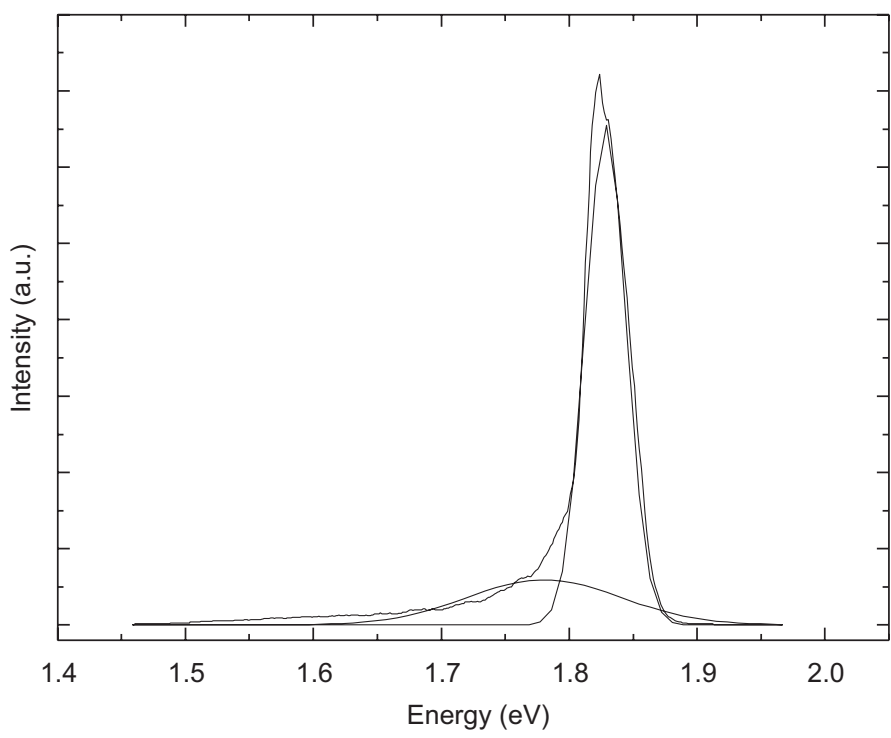

Fig. 2. (a) XRD pattern of 1-D CdSe nanostructures on the sample. (b) PL spectrum obtained from the measurement of the sample's surface at low temperature. The spectrum could be fitted with two Gaussian curves at 1.78 and $1.83 \mathrm{eV}$, respectively.

a

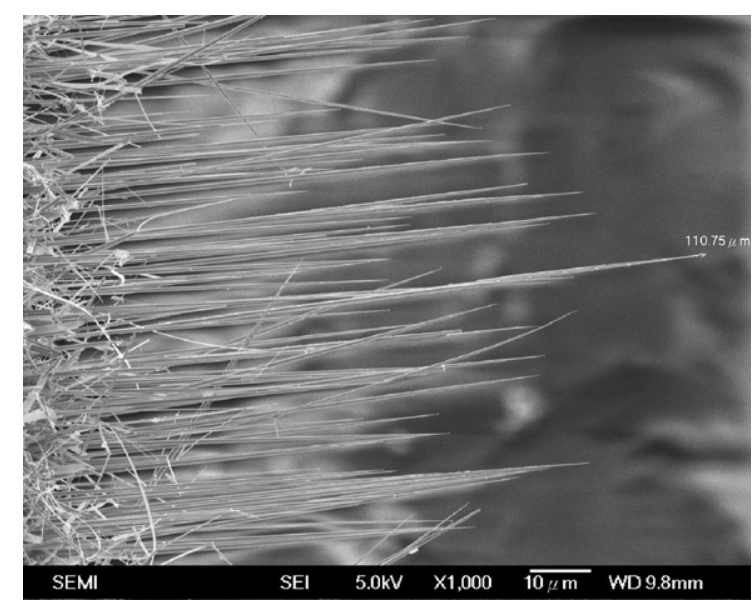

b

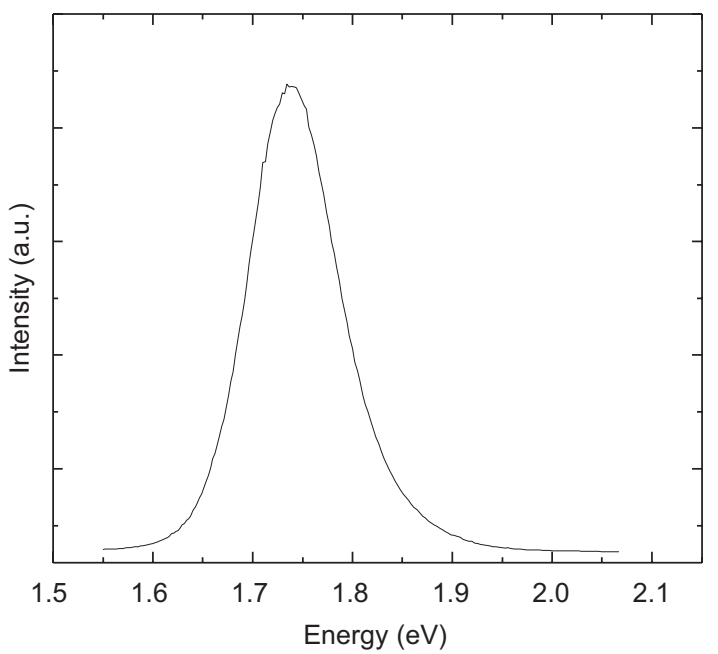

Fig. 3. (a) SEM image of CdSe nanoneedles at the edge of the sample. The length of the middle needle reaches $110.75 \mu \mathrm{m}$. (b) Micro-PL spectrum of individual $\mathrm{CdSe}$ nanoneedle at room temperature.

was found on the top of the nanoneedles grown on the side surface. This result is reasonable as the side surface was not coated with Au prior to the sample growth. The results indicate that the nanoneedles could be grown without the catalyst.

In conclusion, CdSe nanoneedles and other 1-D CdSe nanostructures were grown successfully by MOCVD. The nanoneedles grown at the sample's side surface were grown without using $\mathrm{Au}$ as the catalyst. The nanoneedles could have length over $100 \mu \mathrm{m}$ and are well-aligned and have wurtzite structure. 1-D CdSe nanostructures, such as nanorod, nanowires, and nanobelts, were grown on the sample's surface. It was found that these nanostructures could have either wurtzite or zinc-blende crystal structures and that there are more nanostructures with wurtzite structure than with zinc-blende structure. We also found that increasing thickness of the gold film sputtered on the substrate could better align the nanorods that were grown on the top surface of the substrate.

\section{References}

[1] J. Kong, N.R. Franklin, C. Zhou, M.G. Chapline, S. Peng, K. Cho, H. Dai, Science 287 (2000) 622.

[2] J.S. Jie, W.J. Zhang, Y. Jiang, S.T. Lee, Appl. Phys. Lett. 89 (2006) 133118.

[3] A. Khandelwal, D. Jena, J.W. Grebinski, K.L. Hull, M.K. Kuno, J. Electron. Mater. 35 (2006) 170. 
[4] Y.H. Yang, B. Wang, N.S. Xu, G.W. Yang, Appl. Phys. Lett. 89 (2006) 043108.

[5] P.X. Gao, J. Song, J. Liu, Z.L. Wang, Adv. Mater. 19 (2007) 67.

[6] G.X. Wang, M.S. Park, H.K. Liu, D. Wexler, J. Chen, Appl. Phys. Lett. 88 (2006) 193115.

[7] C.X. Shan, Z. Liu, S.K. Hark, Appl. Phys. Lett. 87 (2005) 163108.

[8] C.X. Shan, Z. Liu, S.K. Hark, Nanotechnology 16 (2005) 3133.
[9] T.L. Chu, S.S. Chu, Solid-State Electron. 38 (1995) 533.

[10] J. Heyd, J.E. Peralta, G.E. Scuseria, R.L. Martin, J. Chem. Phys. 123 (2005) 174101.

[11] U. Lunz, J. Kuhn, F. Goschenhofer, U. Schüssler, S. Einfeldt, C.R. Becker, G. Landwehr, J. Appl. Phys. 80 (1996) 6861.

[12] R.G. Alonso, E.-K. Suh, A.K. Ramdas, N. Samarth, H. Luo, J.K. Furdyna, Phys. Rev. B 40 (1989) 3720. 